\title{
Innovative Composite PDMS Micropump with Electromagnetic Drive
}

\author{
Che-Yi Shen* and Hsien-Kuang Liu** \\ Graduate Institute of Mechanical and Aeronautical Engineering, \\ Feng Chia University, Taichung, Taiwan, Republic of China \\ (Received March 11, 2009; accepted August 18, 2009)
}

Key words: composite, PDMS, valveless, micropump, iron particle, electromagnetic

In this paper, an innovative composite poly-dimethylsiloxane (PDMS) micropump with an electromagnetic drive for biomedical applications that feature low cost and simple assembly is investigated. The developed PDMS micropump based on a reciprocation principle was driven by electromagnetic force that causes cavitation inside a chamber to make fluid flow. A composite PDMS thin film, an iron-particle-dispersed PDMS (IPDP) thin film, is designed, fabricated and driven by electromagnetic force to actuate the micropump. In this work, there are two categories of micropumps including stacked and inlaid types, and each category has four types of micropumps with different geometrical combinations of IPDP and PDMS thin films. The results show that inlaidtype micropumps all have higher flow rates than stacked-type micropumps, and for an inlaid-type-I micropump, the largest IPDP thin film area results in its highest flow rate among the categories. The inlaid-type-I micropump has a maximum flow rate and a backpressure of $1.623 \mathrm{ml} / \mathrm{min}$ and $361.84 \mathrm{~Pa}$, respectively, when applying $30 \mathrm{~V}$ between 6 and $7 \mathrm{~Hz}$ with a low power consumption of $33 \mathrm{~mW}$. As a result of its higher flow rate and new IPDP thin-film design, this kind of full PDMS micropump is appropriate for biomedical applications.

\section{Introduction}

For years, micro-electromechanical system (MEMS) technology, i.e., the miniaturization of sensors and actuators, has been rapidly developing owing to its growing commercial applications. ${ }^{(1)}$ The microfluidic system ${ }^{(2)}$ based on MEMS has been widely investigated. Microfluidic systems have many applications, such as microradiators for laptops and biomedical devices. In microfluidic systems, the micropump is the most important unit for popular fields, such as micro-Total Analysis System $^{(3)}$ (micro-TAS or $\mu$ TAS) and Lab-On-a Chip (LOC). ${ }^{(4)}$ In micropumps, the valve unit is usually studied; however, the inconsistency of the frequency of the valve and the applied frequency may cause damage to the valve. Although the flow rate of

${ }^{*}$ Corresponding author: e-mail: dukesti@pchome.com.tw

${ }^{* *}$ Corresponding author: e-mail: hkliu@fcu.edu.tw 
a valveless micropump may not be the largest among various micropumps, the design of the nozzle/diffuser could simplify the manufacture process and pump structures, and avoid the impairment of valves in the micropump after long-term usage. After the nozzle/diffuser ${ }^{(5)}$ unit was invented, a valveless design without a complicated structure is widely adopted using a simple physical reciprocation principle. Olsson et al.(5) adopted the difference pressure drop between cone-shaped geometries to make the fluid flow. Kim et al. ${ }^{(6)}$ utilized a nozzle/diffuser unit with double chambers that was actuated by piezoelectric disks. Yamahata et al.(7) adopted a magnet embedded in the composite valveless micropump and driven by electromagnetic force. Actuation-type micropumps are the key in fluidic systems, including nonmechanical and mechanical types. Nonmechanical-type micropumps are actuated by the concentration difference between the anions and cations of the fluid or capillary attraction, including electroosmotic, ${ }^{(8)}$ electrochemical, ${ }^{(9)}$ electrophoretic, ${ }^{(10)}$ electro-hydrodynamic (EHD) ${ }^{(11)}$ and magneto-hydrodynamic (MHD) ${ }^{(12)}$ actuations. Mechanical-type micropumps have been extensively developed using various actuation methods, such as thermo-pneumatic, ${ }^{(13)}$ thermal-bubble, ${ }^{(14)}$ electromagnetic, ${ }^{(15)}$ piezoelectric ${ }^{(16)}$ and electrostatic ${ }^{(17)}$ actuations. Although nonmechanical-type micropumps consist of a simpler structure, they usually drive with specific fluids and actuated electrodes. Mechanical-type micropumps with actuators could drive any working fluid with a larger flow rate, which is mainly different from nonmechanical-type micropumps. Cavity chambers are necessary for most mechanical-type micropumps that make the reciprocation to cause fluid flow. Most mechanical-type micropumps show a larger pressure and a faster response than nonmechanical-type micropumps. Previously, the development of microfluidic systems was based on conventional semiconductor materials and techniques originally developed for the integrated circuit industry. Using these techniques for developing microfluidic systems has resulted in not only high cost but also many limitations on fabrication. On the other hand, the recent poly-dimethylsiloxane (PDMS) ${ }^{(18)}$ micromolding technology has been utilized, thereby overcoming such problems. PDMS is one of the most promising polymers used in biomedical devices, such as catheters, tracheoesophageal voice prostheses, finger joints, percutaneous devices, and dentures. ${ }^{(19)}$ PDMS shows good heat stability, oxidation stability, low surface tension, good ventilation and excellent insulation at room temperature; it also shows softness, elasticity and biocompatibility ${ }^{(19,20)}$ at low temperatures; moreover, a PDMS molding with a simple fabrication process and a low cost is very suitable for disposable microfluidic systems, especially for biomedical applications. In addition, the PDMS microfluidic system can be easily integrated with other microfluidic systems within PDMS replication and is applied for large-scale production. It is very easy to fabricate disposable microfluidic systems because the fabrication procedure of the planar geometry of PDMS replication is easy and inexpensive.

In this study, the PDMS is adopted to fabricate the disposable valveless micropump by electromagnetic actuation to investigate its performance. A specially designed IPDP (iron-particle-dispersed PDMS) thin film is fabricated such that a full PDMS micropump, suitable for biomedical applications, can be achieved. Various geometric designs of micropumps are implemented in this work, and the flow rate and back pressure of those 
micropumps driven at various applied voltages and switch frequencies are measured. Two categories of electromagnetic micropumps were demonstrated in terms of their performance.

\section{Experimental Procedures}

\subsection{Principle and design of valveless micropump}

Recently, the valveless micropump has become very popular and its key component is the nozzle/diffuser unit. Figure 1 reveals the principle of the nozzle/diffuser unit in a pump including the supply and pump modes. The volume flow rate $Q_{1}$ is larger than $Q_{2}$ when the chamber is expanded by the actuation of electromagnetic force by an electromagnet, as shown in Fig. 1(a). This is because the inlet fluid speed (larger arrow besides $\left.Q_{1}\right)$ is higher than the outlet speed $\left(Q_{2}\right)$ while the flow passes the same cone-shaped channel but in the reverse direction. A similar function of the nozzle/ diffuser system leads to the volume flow rate $Q_{2}$ being larger than $Q_{1}$ when the chamber collapses, as shown in Fig. 1(b). The result shows that the combination of the above two

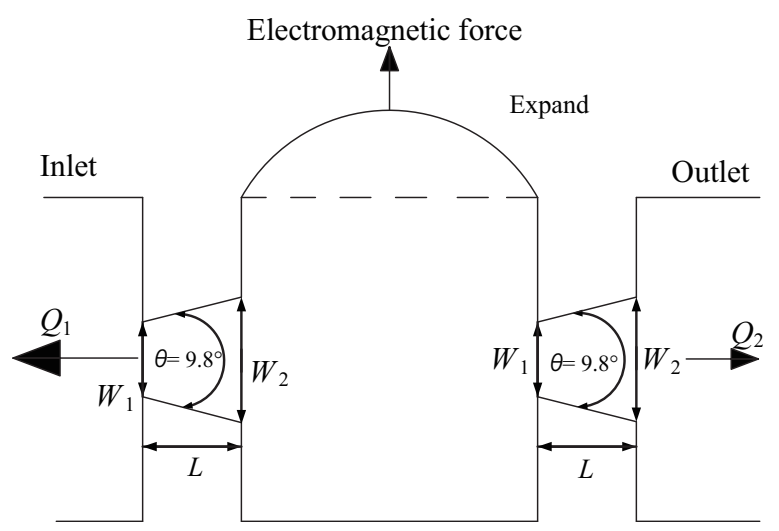

(a)

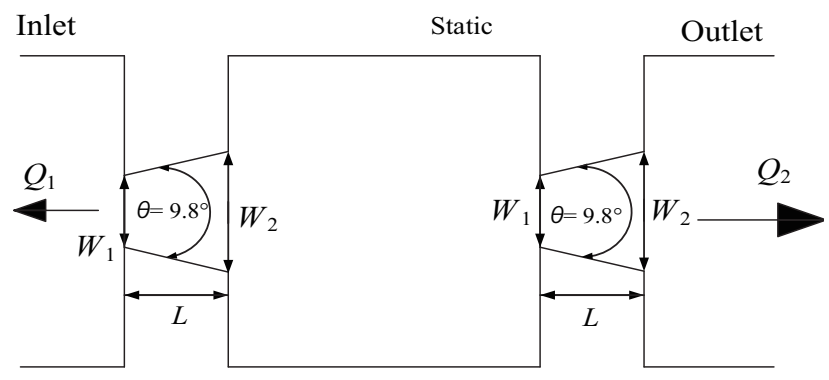

(b)

Fig. 1. Pumping principle: (a) supply mode: $Q_{1}<Q_{2}$; (b) pump mode: $Q_{1}>Q_{2}$. 
modes makes the fluid flow from the inlet to the outlet.

According to the stability map of a diffuser as shown in Fig. 2, ${ }^{(21)}$ the diffuser operates in four different regions depending on the diffuser geometry. In the bistable steady stall (between b-b and c-c lines) and jet flow (above c-c line) regions, the flow performance is poor to extremely poor, as shown in Fig. 2. Under the line a-a, the nostall region, the flow is steady viscous without separation at the diffuser walls and a moderate performance is achieved. In the transitory steady stall region between a-a and b-b lines, the flow is unsteady. The minimum pressure loss and maximum pressurerecovery coefficient $C p$ occur in this region, and hence, the diffuser geometry is designed accordingly. Therefore, the diffuser geometry was designed to be $L / W_{1}=9$ and $2 \theta=9.8^{\circ}$, in which $W_{1}, W_{2}$ and $\mathrm{L}$ are respectively the upper base diameter, lower base diameter, and attitude of the cone-shaped nozzle/diffuser system. Olsson et al. ${ }^{(22)}$ investigated the flow rate and backpressure from slenderness, $L / W_{1}$, and conical angle in 1999. Kim et al. ${ }^{(23)}$ also adopted $L / W_{1}$ and $2 \theta$ between the transitory and steady stall regions.

\subsection{Fabrication of electromagnetic valveless PDMS micropumps}

In this work, two categories of electromagnetic micropumps are studied: one is a stacked-type micropump, as shown in Fig. 3(a), and the other is an inlaid-type micropump, as shown in Fig. 3(b). Both micropumps have the same lower section consisting of a chamber, inlet, outlet and nozzle/diffuser unit. The upper section of the inlaid-type micropump includes an inlaid thin film, in which an iron-particle-dispersed PDMS (IPDP) thin film is embedded in a pure PDMS thin film. The upper section of the

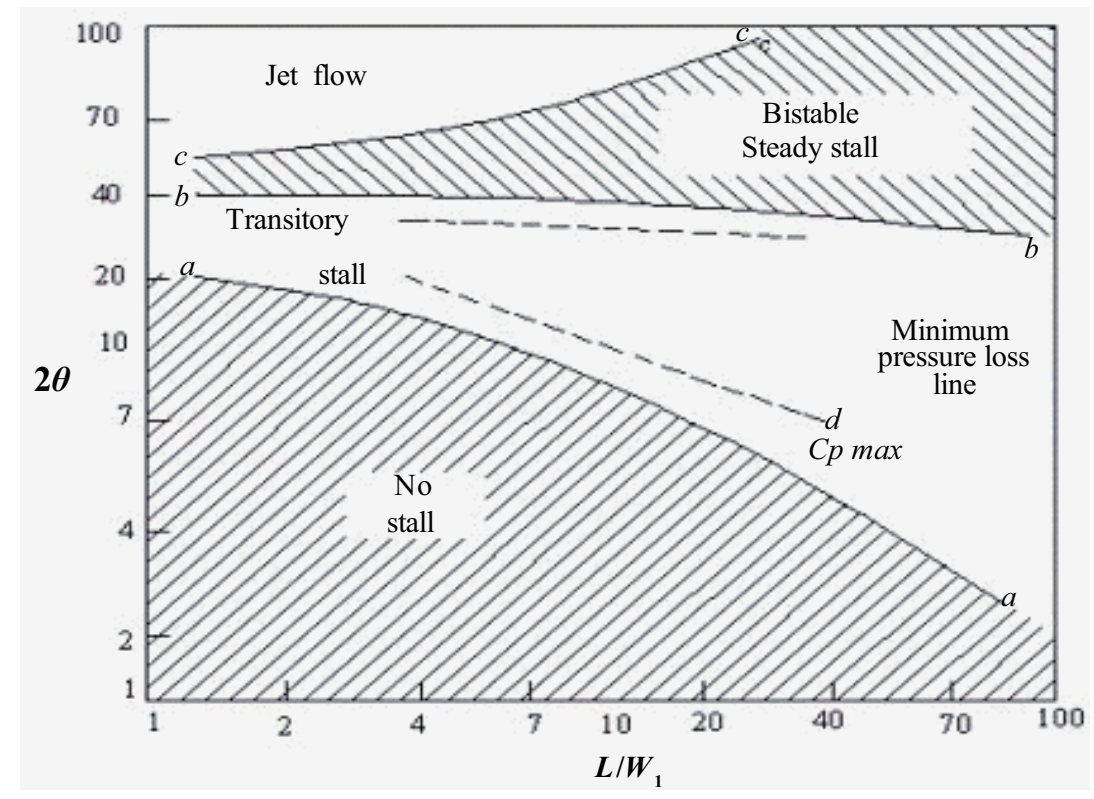

Fig. 2. Relationship between $L / W_{1}$ and $2 \theta$. $^{(20)}$ 


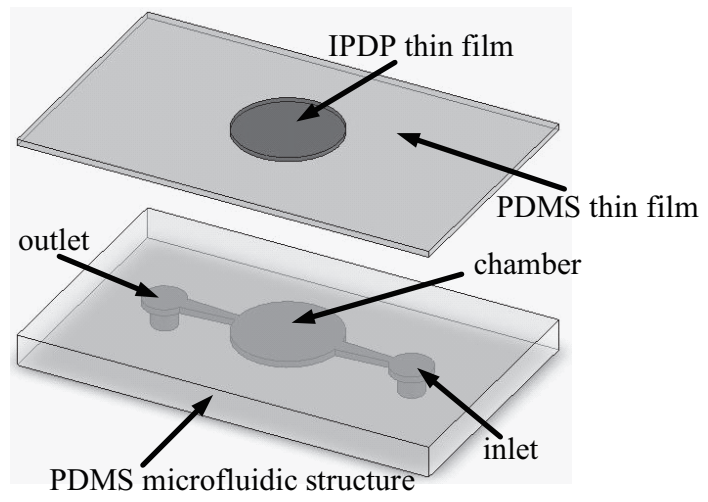

(a)

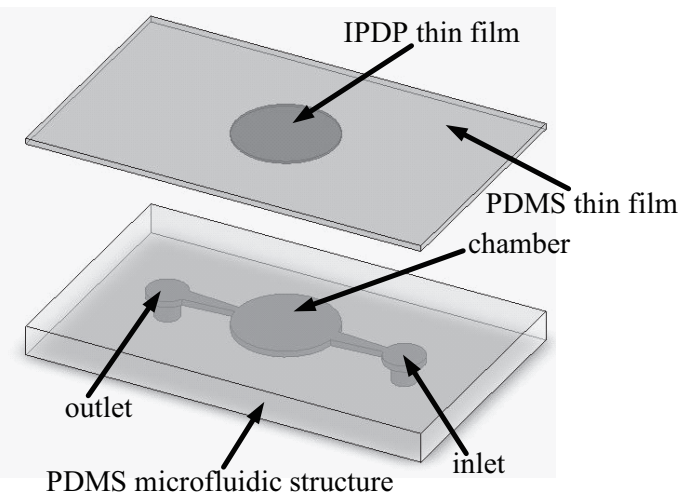

(b)

Fig. 3. 3D perspective overview of two categories of micropumps: (a) stacked type and (b) inlaid type.

stacked-type micropump is composed of stacked thin films, in which an iron-particledispersed PDMS (IPDP) thin film adheres on the pure PDMS thin film. The chamber is $20 \mathrm{~mm}$ in diameter and $500 \mu \mathrm{m}$ in depth. The internal diameters of the inlet and outlet are both $2.5 \mathrm{~mm}$. The side view of all kinds of stacked-type and inlaid-type micropumps with the same chamber diameter size covered by a stacked or inlaid thin film is shown in Fig. 4. The IPDP thin films in stacked-type micropumps I, II, III and IV are respectively $21,10.5,7$ and $3.5 \mathrm{~mm}$ in diameter, with the same thickness of $500 \mu \mathrm{m}$, as shown in Figs. 4(a)-4(d). The same size distribution is applied for inlaid-type micropumps I, II, III and IV, as shown in Figs. 4(e)-4(h), respectively. The difference among the four pump types in each category is in the area of the IPDP thin film. The areas of the IPDP thin films for type I, II, III and IV micropumps are respectively $346.2,86.5,38.5$, and $9.6 \mathrm{~mm}^{2}$. The thickness of the IPDP thin film as well as the PDMS thin film layers for all kinds of micropumps is $500 \mu \mathrm{m}$.

The fabrication process of the two categories of PDMS valveless electromagnetic micropumps is described as follows. For the PDMS microfluidic structure, a polymethylmethacrylate (PMMA) mold with the desired geometry including the inlet, outlet, nozzle/diffuser microchannel and chamber was fabricated, and then, the PDMS (polydimethylsiloxane, Sylgard 184 silicone from Dow Corning, USA) solution mixed with resin and hardener in weight ratio of 10:1 was poured into the PMMA mold. After evacuating in a vacuum chamber for 60 to $90 \mathrm{~min}$, the PDMS polymer was baked at $70^{\circ} \mathrm{C}$ for $180 \mathrm{~min}$ and subsequently cooled to room temperature. Finally, the PDMS microfluidic structure replicas were peeled off the PMMA mold. With this structure, the fabrication processes of stacked-type and inlaid-type micropumps are described respectively in Figs. 5 and 6 . For the inlaid-type micropump, the IPDP thin film was then fabricated as follows. The PDMS solution was well mixed with resin, iron particles (Nippon Shiyaku Kogyo K. K., Japan and the average size of iron particles is $55 \mu \mathrm{m}$ ), 


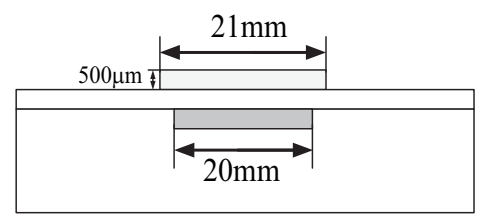

(a) Stacked-type I

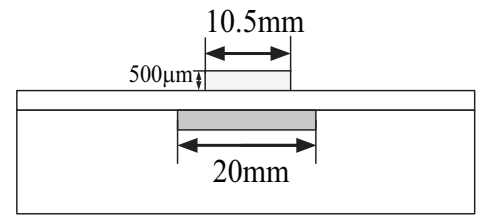

(b) Stacked-type II

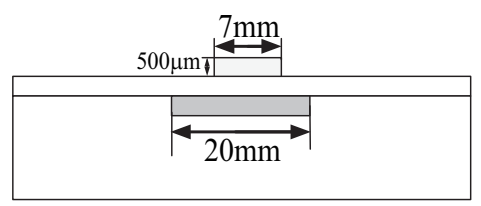

(c) Stacked-type III

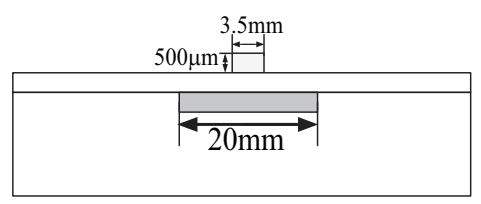

(d) Stacked-type IV

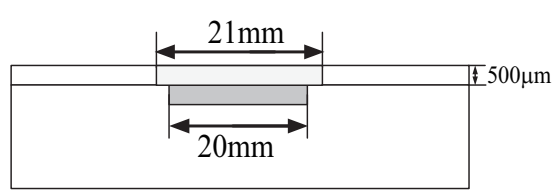

(e) Inlaid-type I

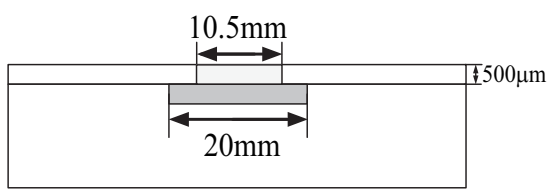

(f) Inlaid-type II

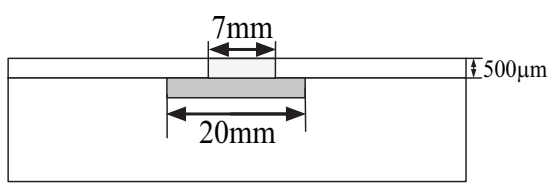

(g) Inlaid-type III

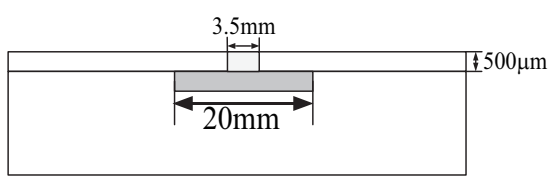

(h) Inlaid-type IV

Fig. 4. Cross-sectional view of eight kinds of micropumps.

and hardener in a weight ratio of 10:10:1 such that iron particles are uniformly dispersed in PDMS; this PDMS mixture was then evacuated in a vacuum chamber for 60 to 90 min. Afterward, the mixture was baked at $80^{\circ} \mathrm{C}$ for $60 \mathrm{~min}$, cooled to room temperature, and finally cut into circles with four different diameters, as shown in Fig. 4. After baking and hardening, PDMS can seal iron powder as the IPDP thin film hermetically and completely. The PDMS solution mixed with resin and hardener in weight ratio of 10:1 was poured around the IPDP thin film at the same thickness. This thin film was evacuated and baked to fabricate the inlaid thin film, and cut at the same length $\times$ width of the microfluidic structure (length $\times$ width, $55 \mathrm{~mm} \times 35 \mathrm{~mm}$ ). The inlaid thin film was attached to the PDMS microfluidic structure by the PDMS solution to achieve a micropump. The stacked-type micropump has a processing different from that of the inlaid-type micropump. Initially, an IPDP thin film was fabricated accordingly. Then, the IPDP thin film was stacked onto the pure PDMS thin film and attached to what by the PDMS solution to obtain a stacked thin film. Similarly, the stacked thin film was attached to the microfluidic structure to achieve a micropump. The fabricated stacked- 
(a)

(b)

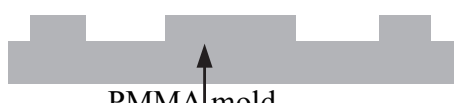

)

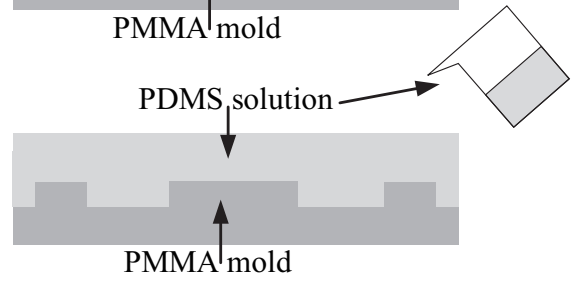

(c) PDMS microfluidic structure

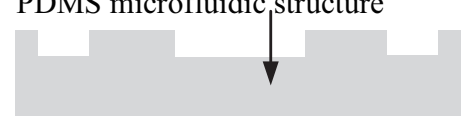

(d)

(e)
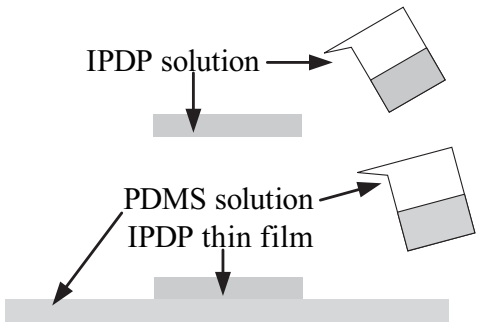

(f)

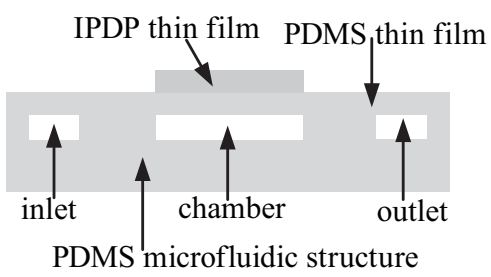

Fig. 5. Fabrication process for PDMS stacked-type micropump: (a) fabricating PMMA mold, (b) pouring the mixed PDMS solution into PMMA mold, (c) producing the microstructure of PDMS, (d) curing the mixture of PDMS solution and iron powder as IPDP thin film, (e) attaching IPDP thin film with specific size to pure PDMS thin film as the stacked thin film and (f) using the stacked thin film with PDMS solution to seal entire microfluidic structure.

(a)

(b)
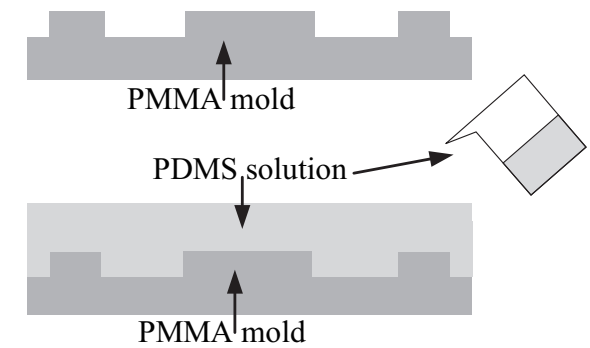

(c) PDMS microfluidic structure

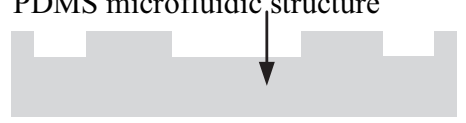

(d)

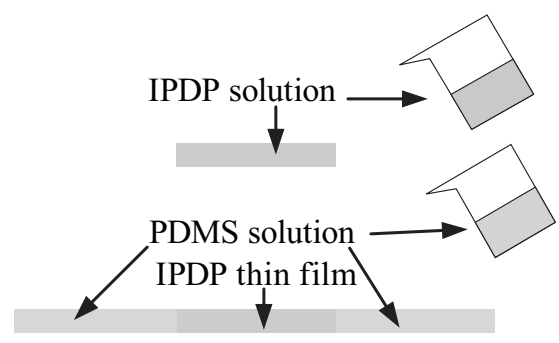

(f)

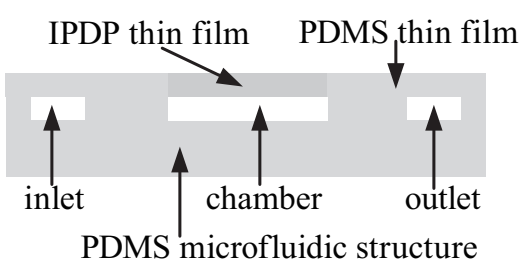

Fig. 6. Fabrication process for PDMS inlaid-type micropump: (a) fabricating PMMA mold, (b) pouring the mixed PDMS solution into PMMA mold, (c) producing the microstructure of PDMS, (d) curing the mixture of PDMS solution and iron powder as IPDP thin film, (e) pouring the PDMS solution around IPDP thin film with specific size and same thickness as the inlaid thin film and (f) using the inlaid thin film with PDMS solution to seal entire microfluidic structure. 
type-I and inlaid-type-I IPDP micropumps are shown in Figs. 7(a) and 7(b), respectively. The chamber that connects to the right outlet and left inlet of the nozzle/diffuser unit is below the black IPDP thin film.

\subsection{Measurement}

To actuate the micropump, the micropump is driven with an electromagnetic force by connecting an electromagnet, a power supply, a combiflex (a device to control the switch on-off of power supply) and a function generator to control the switch frequency of the power supply for actuating the inlaid or stacked thin film. The measurement setups of the water flow rate and back pressure for the micropump are shown in Figs. 8 and 9, respectively. The air gap between the electromagnet and IPDP thin film is always fixed at $500 \mu \mathrm{m}$, which prevents the IPDP thin film contact with the electromagnet, thereby affecting the deflection of the IPDP thin film in the expand mode. The applied voltages on the electromagnet are 20 and $30 \mathrm{~V}$. The frequency range of the applied voltage is from several hertz up to $20 \mathrm{~Hz}$. The backpressure is defined as the height difference between the outlet port and the inlet port. Before actuating, the micropump and water reservoir are placed at the same height to avoid flow by a siphon phenomenon, then the micropump could drive fluid to flow. In terms of the results of Shen and Liu, ${ }^{(24)}$ a micropump with the maximum flow rate occurs at the resonant frequency of the entire

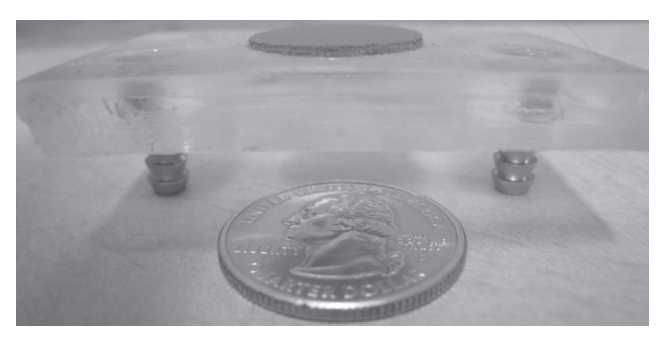

(a)

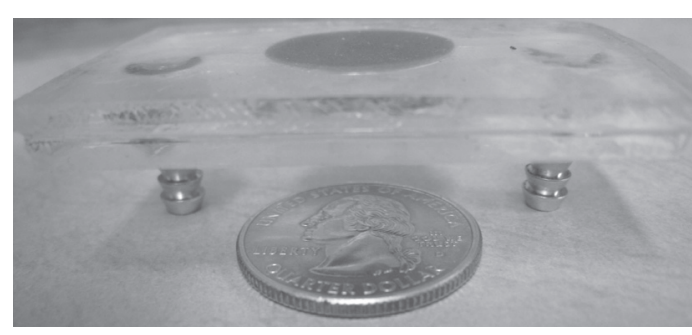

(b)

Fig. 7. Photographs of micropumps: (a) stacked-type I and (b) inlaid-type I.

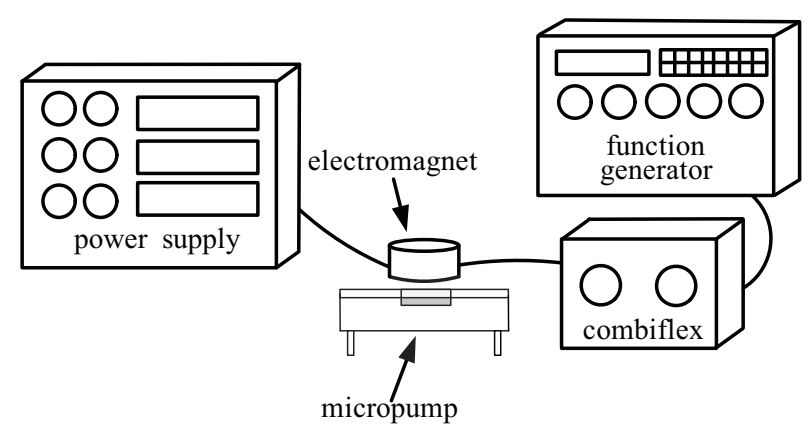

Fig. 8. Experimental setup for measuring flow rate of micropump. 


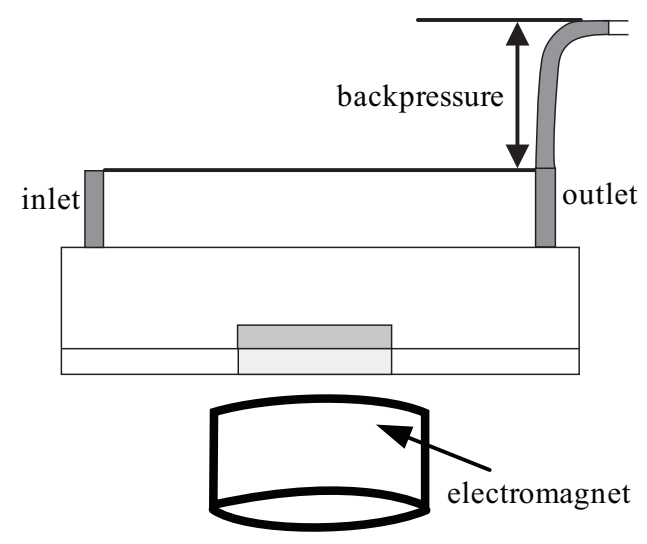

Fig. 9. Experimental setup for measuring the backpressure.

micropump around 15 to $25 \mathrm{~Hz}$ with the maximum deflection and the air bubbles only affect the flow rate of micropump at frequency higher than $90 \mathrm{~Hz}$. For measurement of the electromagnetic force, the experimental setup is shown in Fig. 10. The loss weight above electronic balance is the electromagnetic force when the electromagnet is activated.

\section{Results and Discussion}

\subsection{Flow rate}

Flow rates of the four kinds of inlaid-type micropumps at applied voltages of 20 and $30 \mathrm{~V}$ are shown in Fig. 11. The maximum flow rate of inlaid-type $\mathrm{I}$ is $1.623 \mathrm{ml} / \mathrm{min}$ when applying $30 \mathrm{~V}$ between 6 and $7 \mathrm{~Hz}$. The maximum flow rates of inlaid-types II, III and IV are respectively 1.124, 0.947, and $0.817 \mathrm{ml} / \mathrm{min}$ between 6 and $7 \mathrm{~Hz}$ as well. The flow rates of the four inlaid-type micropumps increase when the applied frequency increases in the range from 0.5 to $7 \mathrm{~Hz}$, and all the maximum flow rates were observed between 6 and $7 \mathrm{~Hz}$ because the resonant frequency of a micropump is close to this range. However, the flow rates of the four inlaid-type micropumps decrease when the applied frequency increases in the range from 7 to $20 \mathrm{~Hz}$. The flow rates of all the inlaid-type micropumps approach zero when the applied frequency is larger than $20 \mathrm{~Hz}$. The flow rate of the micropumps subjected to the applied voltage of $30 \mathrm{~V}$ is larger than that subjected to the applied voltage of $20 \mathrm{~V}$, because the former provides a larger power of $33 \mathrm{~mW}$ (current of power supply, $1.1 \mathrm{~mA})$ than the latter $(22 \mathrm{~mW})$. The inlaidtype-I micropump has the highest flow rate among four kinds of inlaid micropumps; the flow rates of the other micropumps from high to low are in the order of inlaidtype micropumps II, III, and IV. The IPDP thin film of a micropump is actuated by an electromagnetic force; therefore, the area of the IPDP thin film is the key parameter affecting electromagnetic force. According to Maxwell's pulling force ${ }^{(25)}$ and Lorentz force formula, ${ }^{(26)}$ the electromagnetic force $F$ is given by 


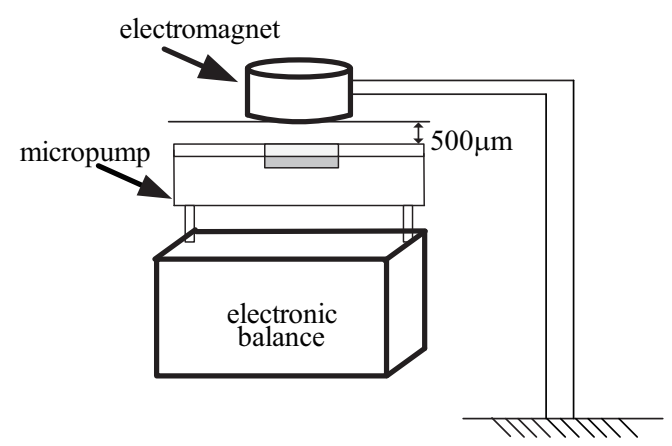

Fig. 10. Experimental setup for measuring the electromagnetic force.

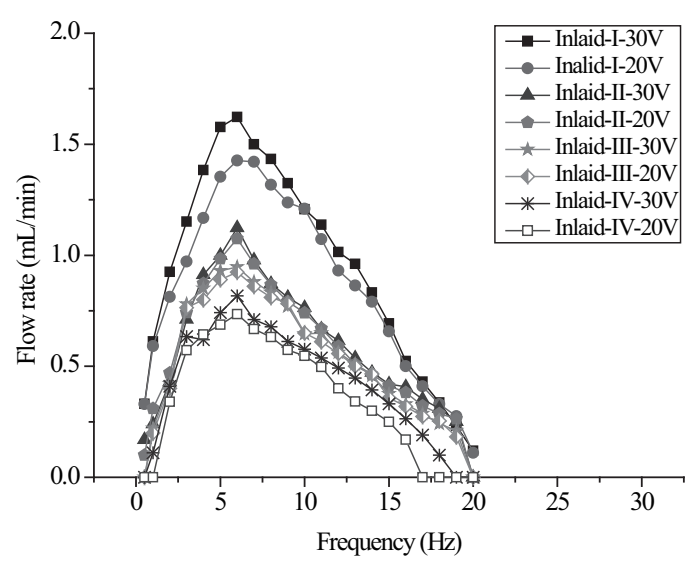

Fig. 11. Relationships between flow rate and frequency for all inlaid-type micropumps at two applied voltages.

$$
F=\frac{B^{2} A}{2 \mu_{0}}
$$

where $F$ is the electromagnetic force, $B$ is the magnetic flux density of the electromagnetic field (by measurement, $B$ is $650 \mathrm{mT}$ when the applied voltage is $20 \mathrm{~V}$ and $B$ is $850 \mathrm{mT}$ when the applied voltage is $30 \mathrm{~V}), A$ is the area of the IPDP thin film (the areas for types I, II, III, and IV are respectively 346.2, 86.5, 38.5, and $9.6 \mathrm{~mm}^{2}$ ), and $\mu_{0}$ is the permeability of a vacuum. In terms of eq. (1), the electromagnetic force is affected by the area of the IPDP thin film at the same magnetic flux density $(B)$ and the permeability of a vacuum $\left(\mu_{0}\right)$. For example, both the inlaid-type micropumps I and II have the same $B$ and $\mu_{0}$ values, but not $A$ (air gap between the electromagnet and the IPDP thin film is always fixed at $500 \mu \mathrm{m}$ ); hence, the electromagnetic force of the 
inlaid-type-I micropump is theoretically four times that of the inlaid-type-II micropump. Depending on the Lorentz force, the larger area provided a higher electromagnetic force at the same electromagnetic field. As shown in Table 1, the measured electromagnetic forces for the inlaid-type micropumps I, II, III, and IV are 178.46, 52.19, 11.18 and $2.75 \mathrm{mN}$, respectively, when the applied voltage is $30 \mathrm{~V}$. Therefore, the inlaid-type-I micropump has the largest flow rate among the four inlaid-type micropumps caused by its largest electromagnetic force.

Theoretically, according to eq. (1) with other parameters remaining constant, the electromagnetic force $(F)$ is proportional to the IPDP area $(A)$. However, for the inlaidtype micropumps II and III with an area ratio of $2.3(9 / 4)$, their measured electromagnetic force ratio of $4.7(52.19 / 11.18)$ is not the same as the area ratio. This indicates that the structure of the micropump would affect the measured electromagnetic force. It is found that when the area ratio of IPDP/chamber (both have the same thickness) is around 0.12 $\left(\left[7^{2} \times \pi / 4\right] /\left[20^{2} \times \pi / 4\right]\right)$, which is the case for the inlaid-type-III micropump, the prediction of electromagnetic force would deviate from eq. (1). It is suggested that the deviation is due to the local nonuniform deflection around a smaller IPDP layer above a larger empty chamber when subjected to electromagnetic force. Therefore, in order to achieve a higher electromagnetic force, the area ratio of IPDP/chamber is suggested to be larger than the critical value of 0.12 . Similarly, as compared with the electromagnetic force at $30 \mathrm{~V}$ between the inlaid-type-I $(178.46 \mathrm{mN})$ and stacked-type-I micropumps (143.72 $\mathrm{mN}$ ) in Table 1, the difference in the force is caused by the structure variation underneath the IPDP layer.

Figure 12 shows the relationship between the flow rate and applied frequency of four kinds of stacked-type micropumps. The maximum flow rate of the stacked-type-I micropump is $1.41 \mathrm{ml} / \mathrm{min}$ when applying $30 \mathrm{~V}$ between 6 and $7 \mathrm{~Hz}$, which is lower than that of the inlaid-type-I micropump. The larger area of the IPDP thin film in the stacked-type-I micropump leads to a larger electromagnetic force as well as a larger power, and results in a flow rate higher than those of the stacked-type micropumps II, III, and IV. According to authors' previous results ${ }^{(24)}$ and other researchers' works, ${ }^{(23,27,28)}$ the maximum flow rate occurred at the resonant frequency of the micropump due to the maximum deflection. As shown in Table 1, the larger area of the IPDP thin film leads to a larger electromagnetic force that results in a higher flow rate due to a larger deflection.

In the comparison of Figs. 11 and 12, in addition to the difference in electromagnetic force shown in Table 1, the flow rate of the stacked-type micropump that is lower than that of the inlaid-type micropump could be attributed to two other factors: stiffness and mass difference. As mentioned in the previous paragraph, the deflection of the film

Table 1

Electromagnetic forces of all micropumps.

\begin{tabular}{lccrc}
\hline & Type I & Type II & \multicolumn{1}{c}{ Type III } & Type IV \\
\hline Inlaid-type (20 V) & $55.03 \mathrm{mN}$ & $14.72 \mathrm{mN}$ & $1.37 \mathrm{mN}$ & $0.4 \mathrm{mN}$ \\
Inlaid-type (30 V) & $178.46 \mathrm{mN}$ & $52.19 \mathrm{mN}$ & $11.18 \mathrm{mN}$ & $2.75 \mathrm{mN}$ \\
Stacked-type (20 V) & $45.1 \mathrm{mN}$ & $11.86 \mathrm{mN}$ & $1.13 \mathrm{mN}$ & $0.33 \mathrm{mN}$ \\
Stacked-type (30 V) & $143.72 \mathrm{mN}$ & $42.11 \mathrm{mN}$ & $9.03 \mathrm{mN}$ & $2.24 \mathrm{mN}$ \\
\hline
\end{tabular}




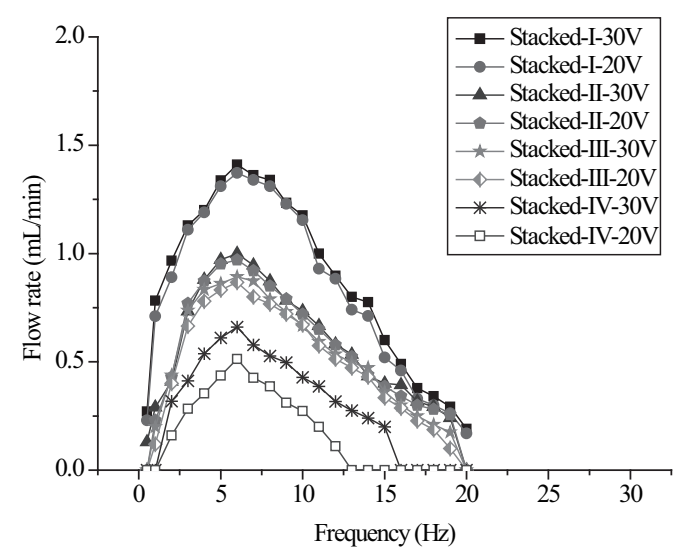

Fig. 12. Relationships between flow rate and frequency for all stacked-type micropumps at two applied voltages.

above the chamber determines the flow rate. Under the same electromagnetic force (IPDP area), the film of the stacked type that consists of both IPDP and PDMS layers has a higher stiffness than the film of inlaid type with only one PDMS layer, thus resulting in a lower deflection as well as a lower flow rate.

Furthermore, the stacked-type micropump with an extra weight of the pure PDMS thin film underneath the IPDP thin film constrains the vibration behavior, thereby limiting the driving effect. For example, although both the inlaid-type-I and stackedtype-I micropumps have IPDP thin films of the same size, under the same driving electromagnetic force, the stacked-type-I micropump needs to drive both the IPDP and PDMS thin films underneath, which are $0.2 \mathrm{~g}$ heavier than that driven by the inlaid-type-I micropump. By Newton's second law, a heavier mass results in a lower acceleration under the same electromagnetic force. The proper integration of this lower acceleration with respect to time would result in a lower vibration speed as well as a lower frequency of both layers above the stacked-type chamber. This could shift its vibration frequency from the resonant frequency. Therefore, the inlaid-type micropump with no additional weight of the pure PDMS thin film has a higher flow rate owing to its higher vibration frequency.

\subsection{Back pressure}

Figures 13 and 14 show the relationships between the back pressure and applied switch frequency of the four kinds of inlaid-type and stacked-type micropumps, respectively. The trends of the backpressure of all micropumps in both Figs. 13 and 14 are similar to those of flow rates shown in Figs. 10 and 11, respectively, i.e., the inlaidtype-I micropump has the highest back pressure and the stacked-type-IV micropump has the lowest back pressure.

For the inlaid-type-I micropump, its back pressure increases with the increase in applied voltage because a higher voltage provides a stronger electromagnetic force. The back pressure also increases with increasing size of the IPDP thin film for similar 


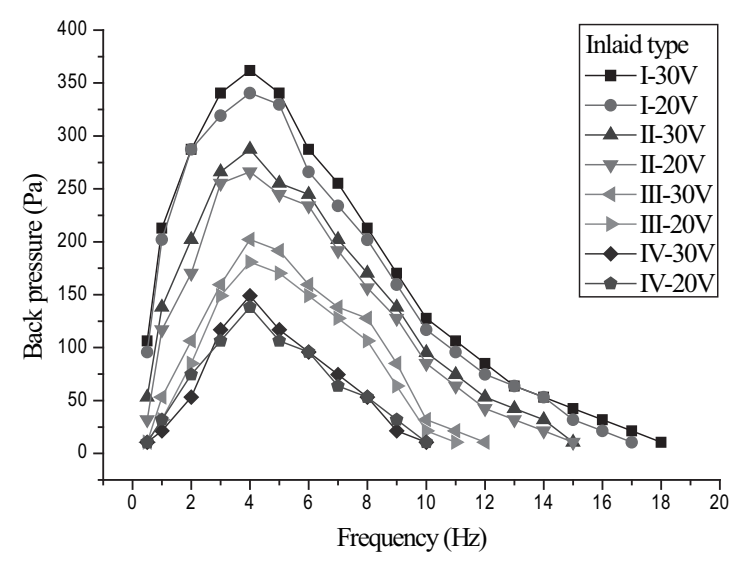

Fig. 13. Relationships between back pressure and frequency for all inlaid-type micropumps.

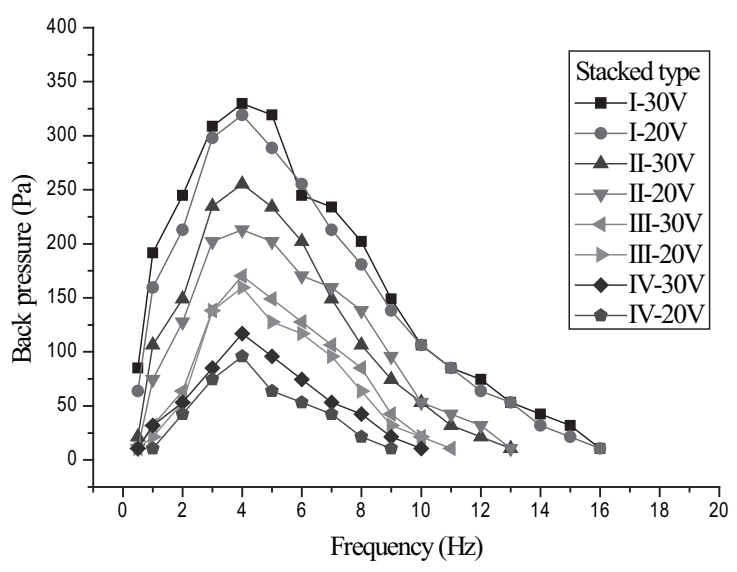

Fig. 14. Relationships between back pressure and frequency for all stacked-type micropumps.

reasons; hence, the back pressure of the inlaid-type-I micropump is larger than that of the inlaid-type-II micropump.

Both the back pressure and flow rate of the inlaid-type micropump are larger than those of the stacked-type micropump. Of all the micropumps, the inlaid-type-I micropump has the highest flow rate and largest back pressure because it has the largest IPDP thin film and the lowest driving inertia that provides the highest driving force for the micropump. Table 2 shows a comparison of the key characteristics of several electromagnetic and piezoelectric micropumps. The former is made of metal and plastic materials, whereas the latter is made of polymers and plastic materials. EsoxPump ${ }^{\mathrm{TM}}$ $\mathrm{V} 01^{(29)}$ is a polymeric implantable pump that has been adapted from the clinical market. Furthermore, Bartels $\mathrm{mP5}^{(30)}$ and ThinXXS MDP1304(31) are positive displacement (the thin film above the chamber moves from its stationary state to expand similarly in 
Table 2

Key characteristics of several micropumps.

\begin{tabular}{|c|c|c|c|c|c|c|c|}
\hline & Pump type & Materials & Dimension & $\begin{array}{l}\text { Pumping } \\
\text { efficiency } \\
\text { (flow rate/ } \\
\text { dimension) }\end{array}$ & Flow rate & $\begin{array}{c}\text { Back } \\
\text { pressure }\end{array}$ & $\begin{array}{c}\text { Power } \\
\text { consumption }\end{array}$ \\
\hline $\begin{array}{l}\text { Esox Pump } \\
\text { V01 }\end{array}$ & $\begin{array}{c}\text { Pressurized } \\
\text { (piezoelectric } \\
\text { actuated) }\end{array}$ & $\begin{array}{l}\text { Steel and } \\
\text { polymer }\end{array}$ & $\begin{array}{c}25 \times 25 \times 7 \\
\mathrm{~mm}^{3}\end{array}$ & $3.2 \times 10^{-4} \mathrm{~min}^{-1}$ & $\begin{array}{c}0.035-1.4 \\
\mu 1 / \min \end{array}$ & NA & NA \\
\hline Bartels mP5(30) & $\begin{array}{c}\text { Positive } \\
\text { Displacement } \\
\text { (piezoelectric } \\
\text { actuated) }\end{array}$ & $\begin{array}{l}\text { Plastic } \\
\text { polyphenyl } \\
\text { sulphon }\end{array}$ & $\begin{array}{c}18 \times 15 \times 3 \\
\mathrm{~mm}^{3}\end{array}$ & $3.7 \mathrm{~min}^{-1}$ & $\begin{array}{c}50-3,000 \\
\mu 1 / \min \end{array}$ & $50 \mathrm{kpa}$ & $45 \mathrm{~mW}$ \\
\hline $\begin{array}{l}\text { ThinXXS } \\
\text { MDP1304 }\end{array}$ & $\begin{array}{c}\text { Positive } \\
\text { Displacement } \\
\text { (piezoelectric } \\
\text { actuated) }\end{array}$ & $\begin{array}{l}\text { Metal and } \\
\text { plastic }\end{array}$ & $\begin{array}{c}26 \times 25 \times 13 \\
\mathrm{~mm}^{3}\end{array}$ & $0.71 \mathrm{~min}^{-1}$ & $\begin{array}{l}6,000 \\
\mu 1 / \min \end{array}$ & $30 \mathrm{kpa}$ & NA \\
\hline Unger et al. ${ }^{(32)}$ & $\begin{array}{c}\text { Peristaltic } \\
\text { (piezoelectric } \\
\text { actuated) }\end{array}$ & $\begin{array}{l}\text { Silicone } \\
\text { rubber }\end{array}$ & NA & NA & $\begin{array}{c}0.141 \\
\mu 1 / \min \end{array}$ & $70 \mathrm{kpa}$ & NA \\
\hline Pan et al. ${ }^{(33)}$ & $\begin{array}{c}\text { Positive } \\
\text { Displacement } \\
\text { (electromagnetic } \\
\text { actuated) }\end{array}$ & $\begin{array}{l}\text { PDMS and } \\
\text { motor }\end{array}$ & $\begin{array}{c}25 \times 10 \times 10 \\
\mathrm{~mm}^{3}\end{array}$ & $0.32 \mathrm{~min}^{-1}$ & $\begin{array}{c}200-800 \\
\mu 1 / \min \end{array}$ & $7.5 \mathrm{kpa}$ & $13 \mathrm{~mW}$ \\
\hline Pan et al..$^{(33)}$ & $\begin{array}{c}\text { Positive } \\
\text { Displacement } \\
\text { (electromagnetic } \\
\text { actuated) }\end{array}$ & $\begin{array}{l}\text { PDMS } \\
\text { and PCB }\end{array}$ & $\begin{array}{c}12 \times 10 \times 5 \\
\mathrm{~mm}^{3}\end{array}$ & $1.67 \mathrm{~min}^{-1}$ & $\begin{array}{c}200-1,000 \\
\mu 1 / \min \end{array}$ & $3.6 \mathrm{kpa}$ & $500 \mathrm{~mW}$ \\
\hline $\begin{array}{l}\text { Grosjean } \\
\text { and Tai }{ }^{(34)}\end{array}$ & $\begin{array}{c}\text { Positive } \\
\text { Displacement } \\
\text { (electromagnetic } \\
\text { actuated) }\end{array}$ & $\begin{array}{l}\text { Polymers } \\
\text { and silicon }\end{array}$ & $\begin{array}{c}18 \times 18 \times 3 \\
\mathrm{~mm}^{3}\end{array}$ & $4.63 \times 10^{-3} \mathrm{~min}^{-1}$ & $4.5 \mu \mathrm{l} / \mathrm{min}$ & $2.1 \mathrm{kpa}$ & NA \\
\hline $\begin{array}{l}\text { IPDP PDMS } \\
\text { Inlaid I pump }\end{array}$ & $\begin{array}{c}\text { Positive } \\
\text { Displacement } \\
\text { (electromagnetic } \\
\text { actuated) } \\
\end{array}$ & $\begin{array}{l}\text { PDMS and } \\
\text { iron powder }\end{array}$ & $\begin{array}{c}55 \times 35 \times 10 \\
\mathrm{~mm}^{3}\end{array}$ & $8.43 \times 10^{-2} \mathrm{~min}^{-1}$ & $\begin{array}{c}1,623 \\
\mu 1 / \min \end{array}$ & $361.84 \mathrm{pa}$ & $33 \mathrm{~mW}$ \\
\hline
\end{tabular}

Fig. 1) piezoelectric micropumps manufactured by two German companies. Unger et al. ${ }^{(32)}$ developed a piezoelectric micropump using a deformable elastomeric membrane

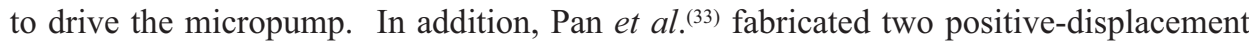
electromagnetic micropumps by PDMS and PCB. Grosjean and Tai ${ }^{(34)}$ at CalTech have fabricated various micropumps using silicon and polymer (Parylene and PDMS). The inlaid-type-I micropump in this study has a larger flow rate than Pan's microcoil-driven pump but a lower back pressure and a lower power consumption. A low back pressure resulting in a higher flow rate is the advantage of our micropump, in that a lower pressure can actuate a higher flow rate. 


\subsection{Pumping efficiency}

Several micropumps with various dimensions and flow rates are listed in Table 2 for comparison. There are two kinds of actuation in the table: piezoelectric and electromagnetic. The difference between piezoelectric and electromagnetic actuations is the applied frequency range. In general, the frequency range for the piezoelectric type is from several $\mathrm{Hz}$ to a couple of $\mathrm{kHz}$ and the frequency range for the electromagnetic type is from $0 \mathrm{~Hz}$ to several hundred $\mathrm{Hz}$. However, the electromagnetic driver can work in a noncontact manner, which may be useful for biomedical applications. Our micropump ranks third among the eight micropumps in terms of flow rate. For MEMS applications, the dimensions of the micropump would play an important role. Therefore, we define a new factor named pumping efficiency by dividing flow rate by the dimensions of the entire micropump (efficiency = flow rate/dimensions). A higher efficiency indicates that a micropump can provide a higher flow rate by unit volume. For further nanoapplications, this would be an important performance index. Judging from the pumping efficiency data in Table 2, the Bartels mP5 micropump has the highest pumping efficiency, although it does not have the highest flow rate. The micropump in this work ranks third among the four electromagnetic pumps in terms of efficiency. The low efficiency of our micropump is probably due to the absorption of driving deflection caused by the low stiffness of the full PDMS micropump. The advantage of our micropump is that iron particles are embedded in PDMS such that the entire micropump is highly biocompatible and can be easily mounted in the human body.

\section{Conclusion}

Novel composite PDMS valveless micropumps were fabricated and actuated by electromagnetic force through the bonding of microfluidic structures with stacked or inlaid PDMS thin films composed of IPDP and pure PDMS thin films; and their performance characteristics were characterized. Micropumps that can be driven by adjustable switch frequency, low applied voltage and low power consumption were successfully demonstrated in this study. The flow rates and back pressures of both stacked-type and inlaid-type micropumps increase with the voltage applied to an electromagnet in a certain frequency range. This electromagnetic actuated micropump is developed with the advantages of high flow rate, low applied voltage, low current and low power consumption. The electromagnetic force and stiffness and mass of the driven layer are three main factors that affect its deflection to affect flow rate and back pressure. With a larger electromagnetic force, a smaller stiffness and a lower mass, inlaid-type micropumps have both a higher flow rate and a higher back pressure than stacked-type micropumps. The inlaid-type-I micropump has the highest flow rate of $1.623 \mathrm{ml} / \mathrm{min}$ and the largest back pressure of $361.84 \mathrm{~Pa}$ among the eight kinds of micropumps. Although the inlaid-type-I micropump shows an intermediate pumping efficiency of $8.43 \times 10^{-2}$ $\min ^{-1}$ among the existing micropumps, considering the volume of the micropump with embedded iron particles in the composite film and a noncontact driving mechanism, it is applicable to biomedical devices. 


\section{Acknowledgements}

The authors of this work are grateful for financial support from the National Science Council in Taiwan under contract NSC 96-2628-E-035-081-MY3.

\section{References}

1 H. G. Craighead: Science 290 (2000) 1532.

2 W. J. Spencer, W. T. Corbett, L. R. Dominguez and B. D. Shafer: IEEE Trans. Sonics Ultrason. 25 (1978) 153.

3 D. R. Reyes, D. Iossifidis, P. A. Auroux and A. Manz: Anal. Chem. 74 (2002) 2623.

4 B. Bilenberg, T. Nielsen, B. Clausen and A. Kristensen: J. Micromech. Microeng. 14 (2004) 814.

5 A. Olsson, E. Stemme and G. Stemme: Sens. Actuators, A 57 (1996) 137.

6 J. H. Kim, C. J. Kang and Y. S. Kim: Microelectron. Eng. 71 (2004) 119.

7 C. Yamahata, F. Lacharme and M. A. M. Gijs: Microelectron. Eng. 78,79 (2005) 132.

8 C. H. Chen and J. G. Santiago: J. Microeletromech. 11 (2002) 672.

9 D. E. Lee, H. P. Chen, S. Soper and W. Wang: Proc. SPIE 4982 (2003) 264.

10 S. K. Hsiung, H. Y. Tseng and G. B. Lee: Tamkang J. Sci. Eng. 8 (2005) 237.

11 J. Darabi, M. M. Ohadi and D. DeVoe: J. Microelectromech. Syst. 10 (2002) 98.

12 J. Jang and S. S. Lee: Sens. Actuators, A 80 (2000) 84.

13 C. G. Cooney and B. C. Towe: Sens. Actuators, A 116 (2004) 519.

14 J. H. Tsai and L. Lin: J. Microelectromech. Syst. 11 (2002) 665.

15 S. Böhm, W. Olthuis and P. Bergveld: Sens. Actuators, A 77 (1999) 223.

16 T. Q. Truong and N. T. Nguyen: J. Microelectromech. Syst. 14 (2004) 632.

17 T. Bourouinay, A. Bosseboeuf and J. P. Grandchamp: J. Microelectromech. Syst. 7 (1997) 186.

18 B. D. DeBusschere, D. A. Borkholder and G. T. A. Kovacs: Solid State Sensors and Actuators Conference (1998) 358.

19 J. Curtis and A. Coals: Biomaterials Science, 2nd ed., ed. B. D. Ratner, A. S. Hoffman, F. J. Schoen and J. E. Lemons (Elsevier Academic Press, San Diego, 2004) Chap. 7.

20 G. Koley, J. Liu, M. W. Nomani, M. Yim, X. Wen and T. Hsia: Mater. Sci. Eng. C 29 (2009) 685.

21 F. M. White: Fluid Mechanics (McGraw-Hill, New York, 1986).

22 A. Olsson, P. Enoksson, G. Stemme and E. Stemme: Micro Electro Mechanical Systems, 1996, MEMS '96, Proceedings, IEEE, 479.

23 J. Kim, C. J. Kang and Y. Kim: Microelectron. Eng. 71 (2004) 119.

24 C. Y. Shen and H. K. Liu: J. Chin. Inst. Eng. 31 (2008) 615.

25 K. Reichert, H. Freundl and W. Vogt: Proc. COMPUMAG (1976) 64.

26 The force produced by a magnetic field, U.K. <http://info.ee.surrey.ac.uk/Workshop/advice/ coils/force.html\#MPF>

27 M. Khoo and C. Liu: Sens. Actuators, A 89 (2007) 259.

28 B. Husband, M. Bu, A. G. R. Evans and T. Melvin: J. Micromech. Microeng. 14 (2004) S64.

29 Esox Technology Corp, CA. <http://dels.nas.edu/ilar_n/ilarjournal/43_3/v4303nolan.shtml>

30 Bartels Mikrotechnik, Dortmund, Germany. <http://www.bartels-mikrotechnik.de>

31 ThinXXS Microtechnology Co, Mainz, Germany. <http://www.thinxxs.com>

32 M. A. Unger, H. Chou, T. Thorsen, A. Scherer and S. R. Quake: Science 288 (2000) 113.

33 T. Pan, S. J. McDonald, E. M. Kai and B. Ziaie: J. Micromech. Microeng. 15 (2005) 1021.

34 C. Grosjean and Y. C. Tai: IEEE Transducers Conf., Korea (1999) 1776. 